\title{
Correlation Between Age Estimates for Elk, Cervus Elaphus, Using Tooth Wear/Eruption Patterns and Counts of Annuli in Tooth Cementum
}

\author{
Rick Rosatte ${ }^{1,4}$, Andrew Silver ${ }^{1}$, Mark Gibson $^{1}$, Bruce Chisholm $^{2}$, and Norm CoOl ${ }^{3}$ \\ ${ }^{1}$ Ontario Ministry of Natural Resources, Wildlife Research and Development Section, Trent University, DNA Building, \\ 2140 East Bank Drive, Peterborough, Ontario K9J 7B8 Canada \\ ${ }^{2}$ Canadian Food Inspection Agency, Edmonton, Alberta, T6H 5T6 Canada \\ ${ }^{3}$ Parks Canada, Elk Island National Park, Fort Saskatchewan, Alberta T8L 2N7 Canada \\ ${ }^{4}$ Corresponding author e-mail: rick.rosatte@ontario.ca
}

Rosatte, Rick, Andrew Silver, Mark Gibson, Bruce Chisholm, and Norm Cool. 2007. Correlation between age estimates for Elk, Cervus elaphus, using tooth wear/eruption patterns and annuli in tooth cementum. Canadian Field-Naturalist 121(2): 214-215.

Two different techniques, (1) tooth wear, tooth eruption patterns and other attributes and (2) counting annuli and seasonal growth zones in the cementum of incisor teeth, were used to estimate the age of Elk, Cervus elaphus, that were relocated from Alberta to Ontario, Canada. Age estimates for Elk ranged from 2 to 20 years, and a significant relationship was found between the ages acquired by the two methods. Critical to acquiring accurate age estimates for Elk were staff with extensive skill with respect to the aging technique utilized.

Key Words: Elk, Cervus elaphus, age estimates, annuli, cementum, tooth wear.

During 1998-2001, 443 Elk (Cervus elaphus) were acquired from Elk Island National Park (EINP), Alberta, Canada, and released in four different areas of Ontario, Canada (Rosatte et al. 2002; Rosatte et al. 2007). Elk were aged according to tooth wear, eruption patterns (deciduous and permanent teeth), colouring and staining of teeth, as well as by comparison to a reference sample of known-age Elk jaws, during processing at EINP (Hudson et al. 2002) (Figure 1). By about two years of age the upper pair of permanent incisiform canines erupt (the ivory) and by about 3 years of age, the 4 pairs of permanent incisors have erupted. The weight of the animal was also considered when estimating the age of Elk at EINP; e.g., the weight of 7-month-old calves, 11/2-year-old, and $\geq 2 \frac{1}{2}$-year-old Elk was approximately $100-120 \mathrm{~kg}, 130-170 \mathrm{~kg}$, and $>180 \mathrm{~kg}( \pm 10 \mathrm{~kg})$, respectively. The weights of the Elk were checked following age estimation to ensure that the age estimate fell into the above age/weight categories.

During 1999-2004, incisor teeth were collected opportunistically from Elk that died in Ontario postrelease. These teeth were aged via counting annuli and seasonal growth zones in tooth cementum (Figure 2). The annuli or rings in the cementum represent yearly growth. By counting the number of annuli in the cementum an estimate of the animals age can be determined (Johnston et al. 1999). The age estimates were compared with those obtained during processing at EINP after the tooth wear age estimates were extrapolated to coincide with the time of death of the Elk in Ontario. Staff involved in aging had many years of experience with the aging technique.

Incisor teeth were extracted (via tooth extractors) from a sample $(n=10)$ of Elk that died in Ontario post-release. Two yearling bulls, for which the age was known, served as controls. Teeth were cleaned of tis-

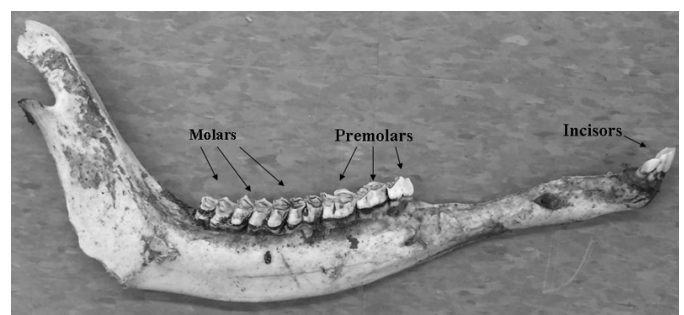

FIGURE 1. Photo of an Elk (Cervus elaphus) mandible showing incisor, premolar, and molar teeth.

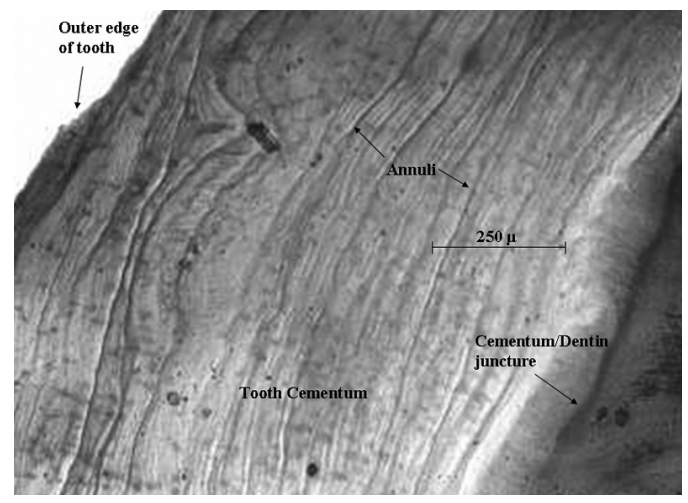

FIGURE 2. Longitudinal section (decalcified, Harris modified hematoxylin stain using bright field illumination) of an incisor tooth (100× magnification) from an Elk (Cervus elaphus) depicting annual growth lines (annuli) in the cementum. Estimated age is 20 years.

sue and soaked in $70 \%$ ethanol for 5 minutes to prevent the transmission of infectious pathogens. They were then preserved in $10 \%$ formalin or frozen at $-20^{\circ} \mathrm{C}$. Teeth were sectioned using an Isomet double-bladed saw (Buehler Isomet, Techmet, Scarborough, Ontario) according to Johnston et al. (1999). Each section (60$100 \mu)$ was mounted on a microscope slide with Per- 
mount and covered with a cover slip. Two microscopic techniques were used to determine an age estimate for each incisor. To distinguish juveniles from adults, the undecalcified, unstained sections were viewed under a compound light microscope $(50 \times$ and $100 \times$ magnification) using polarized light to view seasonal grow zones. In the majority of sections (especially older animals), the annuli were not distinct and not easily discerned under polarized light. These were decalcified (according to Johnston et al. 1999), stained (Harris modified hematoxylin), and placed in $70 \%$ ethanol for 2-3 minutes. Slides were then air-dried, covered with Permount and a cover slip, and examined under the microscope (unpolarized light) to count the number of annuli to obtain an estimate of age (Johnston et al. 1999) (Figure 2).

Age estimates for Elk acquired by tooth wear (and other indicators as noted above) and annuli or seasonal growth zone counts in incisor teeth, ranged between 2 and 20 years of age (Figure 3). The first week of June was used as the birth date for Elk (Hudson et al. 2002). A simple linear correlation and a $t$ statistic (Zar 1974) were used (Statistica 6.0 software, StatSoft, Tulsa, Oklahoma, USA) to measure the relationship and level of significance between tooth wear and counting annuli for age determination in Elk. The correlation was found to be significant $(P<0.0001 ; \mathrm{t}=20.1 ; r=0.9903$; $\left.r^{2}=0.98\right)$. There was a positive slope of 1.09 between the ages acquired by the two methods $(\mathrm{Y}=1.0918$ $x=1.261$ ) (Figure 3).

This study examined the relationship between two methods used to estimate the ages of wild Elk. Tooth wear estimates of age may be inexact as tooth wear could be affected by diet, soil type and substrate (Hudson et al. 2002). However, as the Elk at EINP were in an enclosed (fenced) situation, these variables were fairly constant as their geographical location remained unchanged among years. Therefore, we believe the age estimates acquired by tooth wear (and other attributes that were examined) were fairly accurate. Some authors (Keiss 1969; Hamlin et al. 2000; Hudson et al. 2002) suggest that staining sections of teeth and counting annuli provides the most reliable estimate of age for Elk. Furthermore, Keiss (1969) and Hamlin et al. (2000) only found about a $50 \%$ agreement between tooth eruption/wear and cementum annuli counts and concluded that wear/eruption estimates are unreliable for aging Elk. On the contrary, in our study, although the sample size was small, age estimates for Elk obtained by tooth wear correlated well with those acquired by counting annuli in stained and unstained sections of incisor teeth. We believe that the different result in this study was due to staff expertise with both aging techniques and that this is critical to obtaining accurate age estimates for Elk.

\section{Acknowledgments}

The Ontario Elk restoration and research program is supported by the Ontario Ministry of Natural Resources

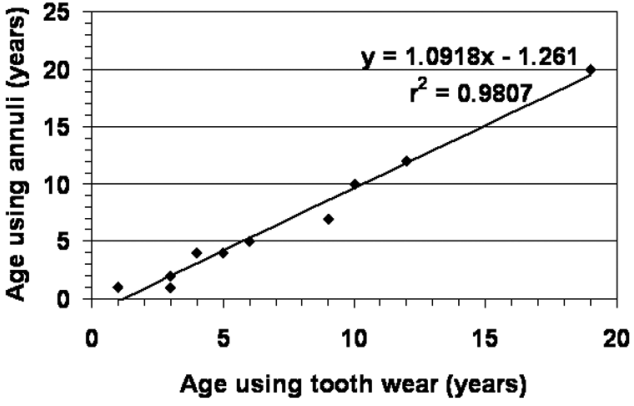

FIGURE 3. Relationship between age of Elk (Cervus elaphus) determined by tooth wear and annual growth lines (annuli) in tooth cementum $(n=10)$.

(OMNR), Wildlife Research and Development Section, Dr. J. Chris Davies, manager, and the OMNR, Wildlife Section, Deb Stetson, manager. Special acknowledgment goes to the staff of the OMNR, Rabies Research and Development Unit, Peterborough, Ontario, to Trent University graduate students, Peterborough, Ontario, to the Canadian Food Inspection Agency, Edmonton, Alberta, and to Parks Canada, EINP, Alberta (especially Rob Kaye), who assisted with the acquisition of teeth for aging and/or the processing of elk at EINP. The manuscript was reviewed by Dr. J. Chris Davies, B. Stevenson, and P. Bachmann, OMNR.

\section{Literature Cited}

Hamlin, K., D. Pac, C. Sime, R. DeSimone, and G. Dusek. 2000. Evaluating the accuracy of ages obtained by two methods for Montana ungulates. Journal of Wildlife Management 64: 441-449.

Hudson, R., J. Haigh, and A. Bubenik. 2002. Physical and physiological adaptations. Pages 199-257 in North American elk: ecology and management. Edited by D. Toweill and J. W. Thomas. Smithsonian Institution Press, Washington, D.C.

Johnston, D. H., D. G. Joachim, P. Bachmann, K. V. Kardong, R. E. A. Stewart, L. M. Dix, M. A. Strickland, and I. D. Watt. 1999. Aging furbearers using tooth structure and biomarkers. Pages 228-243 in Wild Furbearer Management and Conservation in North America. Edited by M. Novak, J. A. Baker, M. E. Obbard, and B. Malloch. [CD Edition] Ontario Fur Managers Federation, Sault Ste. Marie, Ontario.

Keiss, R. 1969. Comparison of eruption-wear patterns and cementum annuli as age criteria in elk. Journal of Wildlife Management 33: 175-180.

Rosatte, R., J. Hamr, B. Ranta, J. Young, and N. Cool. 2002. Elk restoration in Ontario, Canada: Infectious disease management strategy, 1998-2001. Annals of the New York Academy of Sciences 969: 358-363.

Rosatte, R., J. Hamr, J. Young, I. Filion, and H. Smith. 2007. The restoration of Elk (Cervus elaphus) in Ontario, Canada: 1998-2005. Restoration Ecology 15: 34-43.

Zar, J. 1974. Biostatistical analysis. Prentice Hall Inc., Engelwood Cliffs, New Jersey, 620 pages.

Received 6 March 2006

Accepted 14 March 2008 